\title{
TRPV1 Gene Deficiency Attenuates Miniature EPSC Potentiation Induced by Mannitol and Angiotensin II in Supraoptic Magnocellular Neurons
}

\author{
Toru Yokoyama, Takeshi Saito, Toyoaki Ohbuchi, Hirofumi Hashimoto, Hitoshi Suzuki, Hiroki Otsubo, \\ Hiroaki Fujihara, Toshihisa Nagatomo, and Yoichi Ueta \\ Department of Physiology, School of Medicine, University of Occupational and Environmental Health, Kitakyushu 807-8555, Japan
}

\begin{abstract}
The release of arginine vasopressin (AVP) from the magnocellular neurosecretory cells (MNCs) in the supraoptic nucleus (SON) is crucial for body fluid homeostasis. The MNC activity is modulated by synaptic inputs and humoral factors. A recent study demonstrated that an $\mathrm{N}$-terminal splice variant of the transient receptor potential vanilloid type 1 (TRPV1) is essential for osmosensory transduction in the SON. In the present study, we examined the effects of mannitol and angiotensin II on miniature EPSCs (mEPSCs) in the supraoptic MNCs using whole-cell patch-clamp recording in in vitro slice preparation. Mannitol $(60 \mathrm{mM})$ and angiotensin II $(0.1 \mu \mathrm{M})$ increased the frequency of mEPSCs without affecting the amplitude. These effects were attenuated by pre-exposure to a nonspecific TRPV channel blocker, ruthenium red $(10 \mu \mathrm{M})$ and enhanced by pre-exposure to cannabinoid type1 receptor antagonist, AM251 $(2 \mu \mathrm{M})$. Mannitolinduced potentiation of mEPSCs was not attenuated by angiotensin II receptor antagonist, losartan $(10 \mu \mathrm{M})$, indicating independent pathways of mannitol and angiotensin II to the TRPV channels. The potentiation of mEPSCs by mannitol was not mimicked by a TRPV1 agonist, capsaicin, and also not attenuated by TRPV1 blockers, capsazepine $(10 \mu \mathrm{M})$. PKC was involved in angiotensin II-induced potentiation of mEPSCs. The effects of mannitol and angiotensin II on the supraoptic MNCs in trpv1 knock-out mice were significantly attenuated compared with those in wild-type mice counterparts. The results suggest that hyperosmotic stimulation and angiotensin II independently modulate mEPSCs through capsaicin-insensitive TRPV1 channel in the presynaptic terminals of the SON.
\end{abstract}

\section{Introduction}

The release of arginine vasopressin (AVP) from the magnocellular neurosecretory cells (MNCs) in the supraoptic nucleus (SON) is crucial for body fluid homeostasis. The supraoptic MNCs project their axons to the posterior pituitary and secrete arginine vasopressin (AVP) and oxytocin (OXT) into the systemic blood flow. The MNCs in the SON receive a number of different synaptic inputs from the cardiovascular system and osmosensitive areas, and the activity of the MNCs is modulated by excitatory and inhibitory synaptic inputs. In addition to the synaptic inputs, various humoral factors, such as angiotensin II (Okuya et al., 1987; Yang et al., 1992; Nagatomo et al., 1995; Ozaki et al., 2004) modulate the activity of MNCs.

Plasma osmolality is well known to regulate the activity of MNCs (Mason, 1980; Leng et al., 1982; Bourque, 1989). The

Received June 23, 2009; revised Dec. 1, 2009; accepted Dec. 3, 2009.

This study was supported by Grants-in-Aid for Scientific Research on Priority Area No. 18077006 (Y.U.) and Scientific Research (C) No. 20602019 (T.Y.) from the Ministry of Education, Culture, Sports, Science and Technology, Japan, and a Grant-in-Aid for University of Occupational and Environmental Health Research Grant for Promotion of Occupational Health (T.Y.). We thank David Julius (University of California, San Francisco) for providing TRPV1deficient mice and Merck for providing losartan. We are also indebted to Makoto Tominaga and Koji Shibasak (Okazaki Institute for Integrative Bioscience, National Institute for Natural Science, Okazaki, Japan) for valuable technical comments. We also thank Kanako Shoguchi for excellent technical assistance.

Correspondence should be addressed to Dr. Yoichi Ueta, Department of Physiology, School of Medicine, University of Occupational and Environmental Health, 1-1 Iseigaoka, Yahatanishi-ku, Kitakyushu, 807-8555, Japan. E-mail: yoichi@med.uoeh-u.ac.jp.

DOI:10.1523/JNEUROSCI.2986-09.2010

Copyright $\odot 2010$ the authors $\quad 0270-6474 / 10 / 300876-09 \$ 15.00 / 0$ supraoptic MNCs receive synaptic inputs from the organum vasculosum lamina terminals (OVLT), median nucleus of preoptic area $(\mathrm{MnPO})$ and the subfornical organ $(\mathrm{SFO})$. These areas are very sensitive to osmotic changes and regulate body fluid and drinking behavior (Bourque et al., 1994; Richard and Bourque, 1995). In addition to integrative information from the osmosensitive areas, the MNCs are themselves also osmosensitive (Oliet and Bourque, 1992, 1993a). This osmosensitivity is mediated by the osmosensory transduction channel, which is activated by hyperosmotic stimulation and inhibited by hypo-osmotic stimulation (Oliet and Bourque, 1993a,b, 1996). A recent study has demonstrated that an $\mathrm{N}$-terminal splice variant of the transient receptor potential vanilloid type 1 ( $\mathrm{N}$-truncated form of the TRPV1; C-TRPV1) is essential for osmosensory transduction in the supraoptic MNCs (Sharif Naeini et al., 2006). C-TRPV1 knock-out

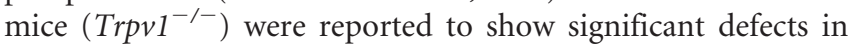
systemic osmoregulation, and their MNCs did not show appropriate bioelectrical response to hyperosmolality and angiotensin II (Sharif Naeini et al., 2006).

Hyperosmotic stimuli and angiotensin II also directly modulate glutamatergic inputs to the supraoptic MNCs by acting on the presynaptic terminals (Inenaga et al., 1997; Ozaki et al., 2004). Our previous study demonstrated that angiotensin II potentiated the miniature EPSCs (mEPSCs) into the supraoptic MNCs via angiotensin type 1 (AT1) receptors (Ozaki et al., 2004). To our knowledge, there is no information on whether the TRPV channels mediate the osmosensitivity and angiotensin II sensitivity in 
the presynaptic terminals of the supraoptic MNCs. Furthermore, little is known about how AT1 receptor activation leads to potentiation of mEPSCs in the presynaptic terminals. In the present study, we examined the effects of TRPV channel blockers on hyperosmotic stimulation- and angiotensin II-induced increases of mEPSCs in the supraoptic MNCs. We also examined signal transduction activated by AT1 receptors in the presynaptic terminals of the supraoptic MNCs.

\section{Materials and Methods}

Animals. Experiments were performed in male Wistar rats weighting $100-200 \mathrm{~g}$, male TRPV1 gene knock-out mice (Trpv1 $\left.{ }^{-/-}, \mathrm{KO}\right)$ and their counterparts (C57BL/6, wild-type) weighting $20-25$ g. Trpv1 $1^{-/}$mice were a kind gift from Dr D. Julius (University of California, San Francisco). Trpv1 $1^{-1-}$ mice were generated as described previously (Caterina et al., 2000). All procedures described in the present study were performed in accordance with guidelines on the use and care of laboratory animals as set out by the Physiological Society of Japan and under the control of the Ethics Committee of Animal Care and Experimentation, University of Occupational and Environmental Health, Japan.

Slice preparations. Rats and mice were killed by decapitation. We ensured the absence of gross contusion and hemorrhage after removal of each brain from the skull. The brains were rapidly removed and cooled in a perfusion medium at $4^{\circ} \mathrm{C}$ for $1 \mathrm{~min}$. A block containing the hypothalamus was cut and glued to the stage of a vibratome-type slicer (DSK Linearslicer PRO7). After careful removal of the meninges, coronal slices (150 $\mu \mathrm{m}$ thick) containing the SON were cut as described previously (Nagatomo et al., 1995). The slices were carefully trimmed with a circular punch (inner diameter $1.8 \mathrm{~mm}$ ) and preincubated in the perfusion medium at room temperature for at least $1 \mathrm{~h}$, after which they were transferred to the recording chamber.

Solutions and drugs. The perfusion medium contained the following (in mM): $124 \mathrm{NaCl} ; 5 \mathrm{KCl} ; 1.24 \mathrm{KH}_{2} \mathrm{PO}_{4} ; 2 \mathrm{CaCl}_{2} ; 25.9 \mathrm{NaHCO}_{3}$; and 10 glucose. For $\mathrm{Ca}^{2+}$-free solution, $\mathrm{Ca}^{2+}$ was replaced by $\mathrm{Mg}^{2+}$ and the osmolality was adjusted by lowering the $\mathrm{Na}^{+}$concentration. The $\mathrm{pH}$ was adjusted to 7.3, and the osmolality of all solutions ranged between 298 and $303 \mathrm{mOsmol} / \mathrm{kg}$. The perfusion medium for the mice slice preparation was modified by increasing osmolality to $312 \mathrm{mOsmol} / \mathrm{kg}$, according to the natural basal serum osmolarity observed in this strain of mice (Stachniak and Bourque, 2006). For hyperosmotic stimulation, mannitol was added to the perfusion medium. Angiotensin II and [Pmp1, $\operatorname{Tyr}(\mathrm{Me}) 2]$-Arg8-vasopressin (PMP-AVP) were purchased from Peptide Institute (Osaka, Japan); tetrodotoxin (TTX) was obtained from Sankyo Co.. Losartan was kindly provided by Merck. Ruthenium red, capsaicin, capsazepine, SB366791, atosiban, DL-fluorocitric acid and bis-(oaminophenoxy)- $N, N, N^{\prime}, N^{\prime}$-tetraacetic acid (BAPTA) were purchased from Sigma-Aldrich, bisindolylmaleimide I and thapsigargin from Calbiochem. AM251 was purchased from Wako. $N$-(4-Tetrarybutylphenyl)-4(3-chloropyridin-2-yl)tetrahydropyrazine-1(2H)-carboxamide (BCTC) was purchased from Biomol. For stock solution, angiotensin II, TTX, losartan, ruthenium red, PMP-AVP and atosiban were each dissolved in distilled water and then dissolved into working solution, with the final concentration of the solvents not being $>0.1 \%$. Capsaicin, capsazepine, SB366791, bisindolylmaleimade I, thapsigargin BCTC and AM251 were each dissolved in dimethyl sulfoxide (DMSO). The final concentration of DMSO in the perfusion medium was $<0.05 \%$. All solutions used in this experiment were bubbled with a mixture of $95 \% \mathrm{O}_{2}-5 \% \mathrm{CO}_{2}$. The pipette solution used in the recording electrodes contained the following (in mM): $140 \mathrm{~K}$-gluconate; $1 \mathrm{MgCl}_{2}, 1 \mathrm{CaCl}_{2} ; 10$ EGTA; and ATP Mg-2 (pH 7.3 with Tris base). TTX was present in all experiments except for $\mathrm{Ca}^{2+}$. free solution.

Whole-cell recordings and data analyses. The slices were fixed in a recording chamber as described previously (Kabashima et al., 1997). Briefly, the slices were placed onto a glass-bottomed chamber and fixed with a grid of parallel nylon threads supported by a U-shaped stainless steel weight. The volume of the recording chamber was $1 \mathrm{ml}$ and the perfusion rate was $1.4 \mathrm{ml} / \mathrm{min}$. The solution level was kept constant by a low-pressure aspiration system. To identify magnocellular neurons in the SON, we used an upright microscope (BX-50, Olympus) with Nomarski optics $(400 \times)$. Drugs were applied to the slice preparation by switching the perfusion solution using a two-way valve (HV 4-4, Hamilton). The dead space washing time was excluded from calculations.

The electrodes used in this study were triple-pulled with a puller (P-87, Sutter Instrument Co.) from a glass capillary, and had a final resistance of 5-9 $\mathrm{M} \Omega$ when filled with the electrode solution. Electrophysiological recordings were performed at $32-33^{\circ} \mathrm{C}$. Whole-cell recordings were made from microscopically identified SON neurons in the upper surface layers of the slices. Recordings of postsynaptic currents began 5 min after membrane rupture when the current reached a steady state. Currents and voltages were recorded with an EPC-10 amplifier (HEKA). Signals were filtered at $3 \mathrm{kHz}$, digitized at $1 \mathrm{kHz}$ with an analog-to-digital converter (MacLab/v. 3.5), and stored on the hard disk of a personal computer. For quantitative analysis of the synaptic currents, only the AC components (using a $1 \mathrm{~Hz}$ high pass filter) were used for analysis with software (AxoGraph V.3.6.1, Molecular Devices). Spontaneous events were automatically screened using an amplitude threshold of $15 \mathrm{pA}$ and then were visually accepted or rejected based on the rise time and decay time. Recordings included for data analysis were collected during periods of stable series resistance (10-20 M $\Omega$ with no compensation).

When using the perfusion medium and the pipette solution indicated above, mEPSCs and miniature IPSCs (mIPSCs) were discriminated by changing the holding potential from $-70 \mathrm{mV}$ to $-20 \mathrm{mV}$ (Kabashima et al., 1997). At $-70 \mathrm{mV}$, virtually pure mEPSCs were recorded, which were completely abolished by application of 6-cyano-7-nitroquin-oxaline2,3-dione (CNQX; a blocker of non-NMDA receptors), while at -20 $\mathrm{mV}$, pure mIPSCs were recorded, which were completely abolished by application of picrotoxin (a blocker of $\mathrm{GABA}_{\mathrm{A}}$ receptor-gated $\mathrm{Cl}^{-}$ channels). Therefore, the holding potentials of $-70 \mathrm{mV}$ and $-20 \mathrm{mV}$ were used to analyze mEPSCs and mIPSCs, respectively, throughout the experiments.

Statistical analysis. Data are expressed as mean \pm SEM with $n$ representing the number of neurons tested. Differences between two groups were examined for statistical significance using the paired $t$ test and between multiple groups by one-way ANOVA. A $p$ value $<0.05$ denoted the presence of a statistically significant difference.

\section{Results}

Spontaneous synaptic currents were recorded from a total of 209 MNCs that were identified microscopically in thin punch-out SON slice preparations from 89 rats and 20 mice. As reported previously (Kabashima et al., 1997), EPSCs and IPSCs were insensitive to the $\mathrm{Na}^{+}$channel blocker TTX $(1 \mu \mathrm{M})$, indicating that spontaneous EPSCs and spontaneous IPSCs recorded from the thin punch-out slice preparations are miniature EPSCs and miniature IPSCs that reflect spontaneous quantal release of glutamate and GABA, respectively. DMSO alone had no significant effect on frequency $(102 \pm 10 \%$ of control) or amplitude $(102 \pm 6.2 \%$ of control) of mEPSCs $(n=4)$.

\section{Effects of hyperosmotic stimulation and angiotensin II on mEPSCs}

Figure $1 A$ shows representative mEPSC recordings and the effects of mannitol and angiotensin II. Mannitol (60 mM)-induced hyperosmotic stimulation $(360 \mathrm{mOsmol} / \mathrm{kg}$ ) and the application of angiotensin II $(0.1 \mu \mathrm{M})$ increased the frequency of mEPSCs. The amplitude of mEPSCs was not affected by mannitol or angiotensin II. The averaged values of frequency and amplitude of mEPSCs during the control period and after drug application were compared. Recordings included for the data analyses were collected over $3 \mathrm{~min}$, before drug application (control) and $5 \mathrm{~min}$ after starting drug application (mannitol and angiotensin II). To confirm the stability of the preparations under whole-cell recording, we also recorded from untreated cells for identical time in- 
terval (No treatment group; frequency $98.9 \pm 5.3 \%$ of control, amplitude $100 \pm$ $2.4 \%$ of control, $n=7$ ). Mannitol significantly increased the frequency of $\mathrm{mEP}$ SCs $(185 \pm 14 \%$ of control, $n=8, p<$ $0.01)$, but did not alter the amplitude of mEPSCs ( $99.1 \pm 1.8 \%$ of control, $n=8)$. Similarly, angiotensin II increased the frequency of mEPSCs $(156 \pm 13 \%$ of control, $n=8, p<0.01)$, but not the amplitude (103 $\pm 2.1 \%$ of control, $n=8)$ (Fig. 1C). To exclude the possible effect of postsynaptic activation on the presynaptic input, BAPTA was added to the pipette solution. A similar potentiation of mEPSCs by mannitol and angiotensin II was observed when the BAPTA (10 mM)containing pipette solution was used (Fig. $1 C)$. The frequency increased significantly (mannitol: $180 \pm 12 \%$ of control, $n=8$, $p<0.01$; angiotensin II: $160 \pm 11 \%$ of control, $n=9, p<0.01)$, but not the amplitude (mannitol: $100 \pm 1.8 \%$ of control, $n=8$; angiotensin II: $106 \pm 1.9 \%$ of control, $n=9$ ). Thus, hyperosmotic stimulation and angiotensin II increase the frequency of mEPSCs presynaptically. In contrast to the effect on the mEPSCs, application of mannitol ( $60 \mathrm{~mm})$ did not affect mIPSCs (frequency $96.9 \pm 3.6 \%$, amplitude $96.8 \pm 1.7 \%$ of control, $n=10$ ).

\section{Effects of TRPV blockers on hyperosmotic stimulation- and angiotensin II-induced potentiation of mEPSCs}

To examine whether the effects of mannitol and angiotensin II are mediated by TRPV channels, we used $10 \mu \mathrm{M}$ ruthenium red, a nonspecific TRPV channel blocker. Figure $1 B$ shows representative examples of the effects of ruthenium red. Pre-exposure to ruthenium red attenuated the potentiation of mEPSCs by mannitol $(60 \mathrm{~mm})$ and angiotensin II $(0.1 \mu \mathrm{M})$. Figure $1 C$ shows a summary data for the effects of ruthenium red on the amplitude and frequency. Ruthenium red almost completely abolished the mannitol- and angiotensin II-induced increase in mEPSC frequency, but had no effect on the amplitude of mEPSCs (mannitol: frequency $106 \pm 8.6 \%$, amplitude $103 \pm 3.4 \%$ of control, $n=6$; angiotensin II: frequency $101 \pm$ $6.1 \%$, amplitude $96.5 \pm 1.8 \%$ of control, $n=13$ ). These results suggest the possible involvement of TRPV channels in both hyperosmotic stimulation- and angiotensin II-induced potentiation of the mEPSCs.

Pharmacological characterizations of TRPV1 channel in hyperosmotic stimulation-induced potentiation of mEPSCs To determine whether TRPV 1 channels mediate the potentiation of mEPSCs, we examined the effects of capsaicin, a TRPV1 channel agonist, and capsazepine, a TRPV1 channel selective
A

(a)

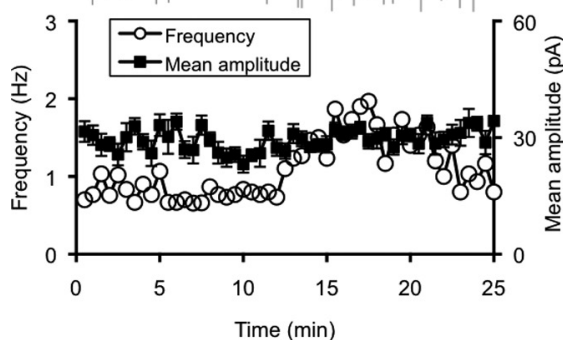

B

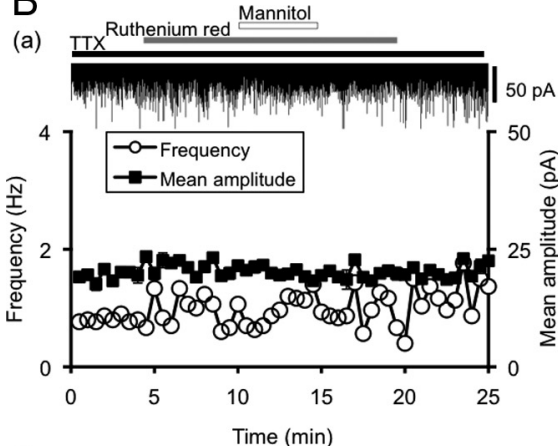

C

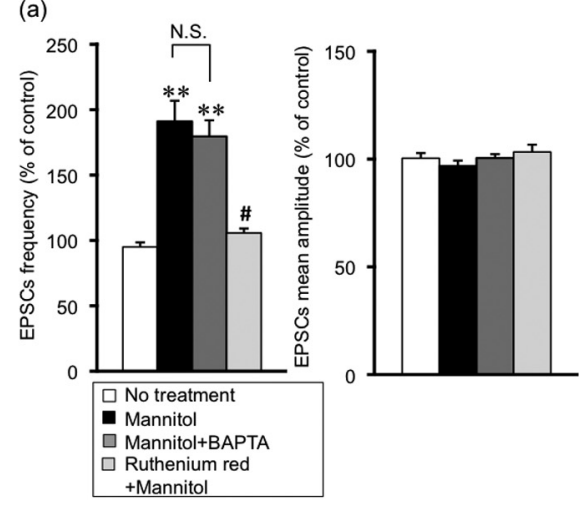

(b)

TTX Angiotensin II
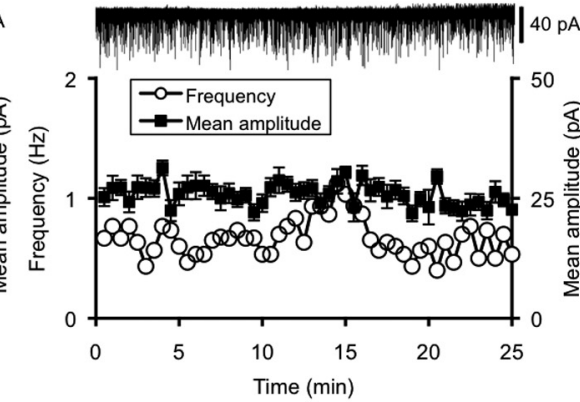

(b) $\pi x^{\text {Ruthenium red Angiotensin II }}$

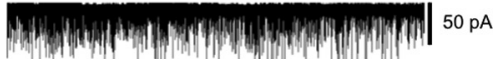

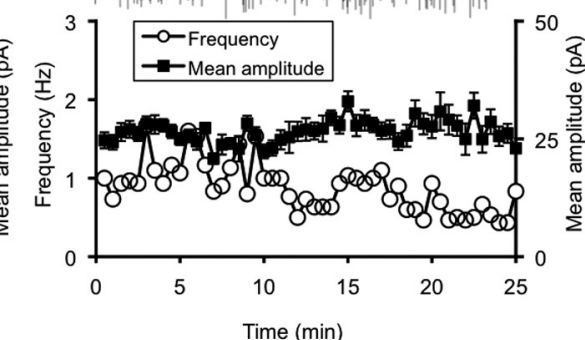

(b)

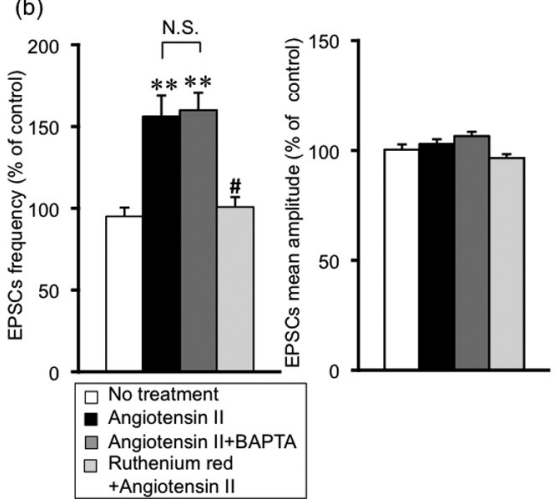

Figure 1. Effects of mannitol and angiotensin II on mEPSCs in the SON. A, Representative examples of mannitol (a) $(60 \mathrm{~mm}, 360$ $\mathrm{m} 0 \mathrm{smol} / \mathrm{kg})$ and angiotensin II (b) $(0.1 \mu \mathrm{M})$ on $\mathrm{mEPSCS}$. EPSCs were recorded in the presence of TTX (1 $\mu \mathrm{M})$. The holding potential was $-70 \mathrm{mV}$. Plots of frequency are single measurements, whereas plots of amplitude are mean \pm SEM over 30 s. $\boldsymbol{B}$, Representative examples of the effects of a nonspecific TRPV1 blocker, ruthenium red (10 $\mu \mathrm{M})$ on mannitol $(\boldsymbol{a})$ - and angiotensin II (b)induced potentiation of $\mathrm{mEPSC}$. Mannitol- and angiotensin II-induced potentiation of $\mathrm{mEPSC} s$ was suppressed by ruthenium red. C, Summary data for the effects of ruthenium red on the frequency and amplitude of mEPSCs. $\boldsymbol{a}$, Frequency (left) and amplitude (right) of mEPSCs. No treatment $(n=7)$, mannitol $(n=8)$, mannitol plus BAPTA $(n=8)$ and mannitol plus ruthenium red $(n=$ 6), respectively. $\boldsymbol{b}$, No treatment $(n=7)$, angiotensin II $(n=8)$, angiotensin II plus BAPTA $(n=9)$ and angiotensin II plus ruthenium red $(n=13)$. BAPTA in the pipette solution did not affect the potentiation of $\mathrm{mEPSC}(\mathrm{s}$ by mannitol and angiotensin II. Data are mean \pm SEM. ${ }^{* *} p<0.01$, compared with no treatment. ${ }^{*} p<0.01$, compared with mannitol or angiotensin II.

blocker. Application of capsaicin $(10 \mu \mathrm{M})$ did not significantly influence mEPSCs (frequency $96.7 \pm 6.4 \%$, amplitude $105 \pm$ $2.9 \%$ of control, $n=9$ ) (Fig. $2 A$ ). The potentiation of mEPSCs by mannitol was not suppressed by pre-exposure to capsazepine (10 $\mu \mathrm{M})$. The frequency increased significantly without affecting the mean amplitude (frequency: $167 \pm 16 \%$ of control, $n=8, p<$ 0.01 , amplitude $99.5 \pm 1.9 \%, n=8$ ) (Fig. $2 B, C$ ). Since the TRPV1 channel selective blockers, SB366791 and BCTC, are known to inhibit TRPV1 channel activation induced by $\mathrm{pH}$ or temperature, we also examined whether these drugs inhibit the potentiation of mEPSCs by mannitol. However, the potentiation of mEPSCs by mannitol was not suppressed by pre-exposure to 
A
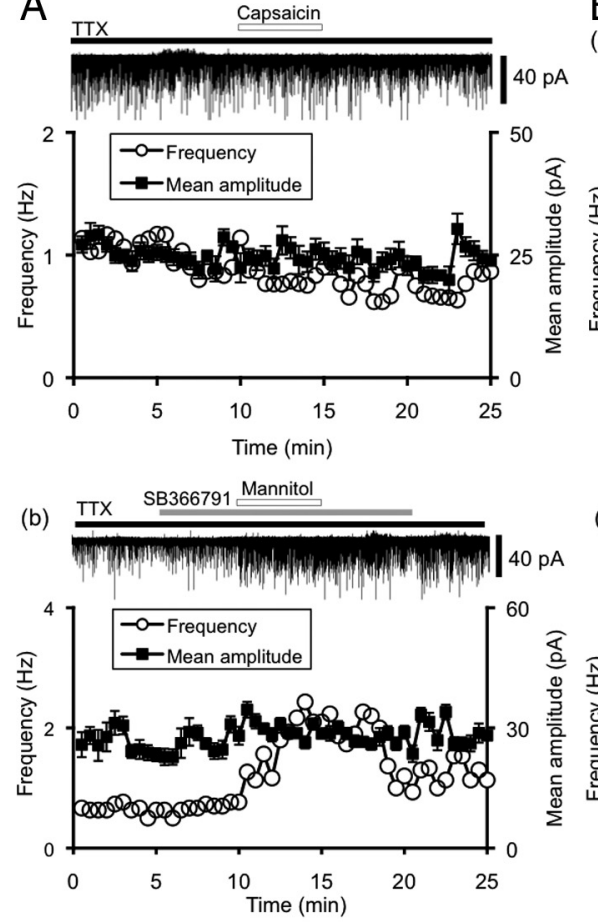

C

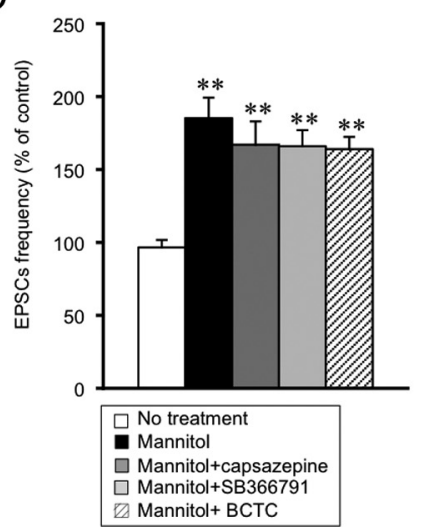

B

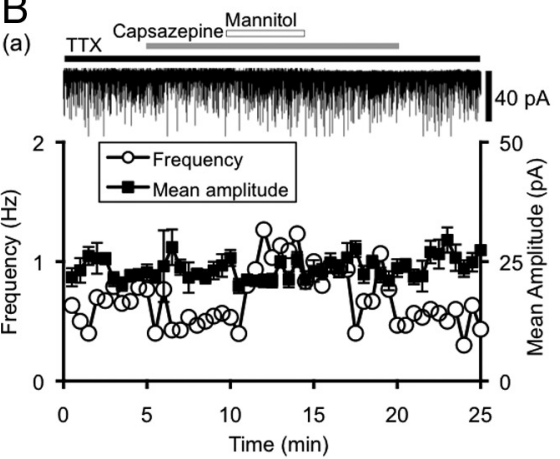

(c)

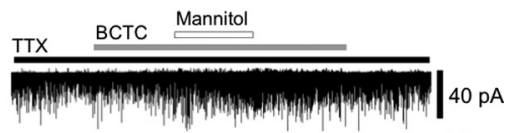

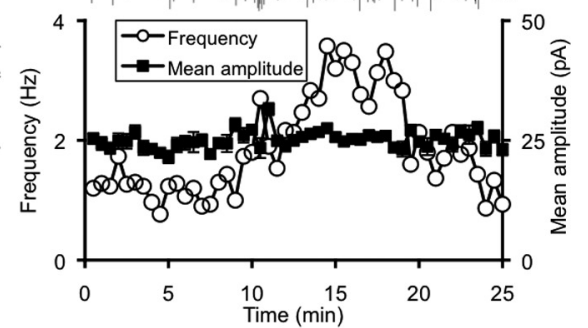

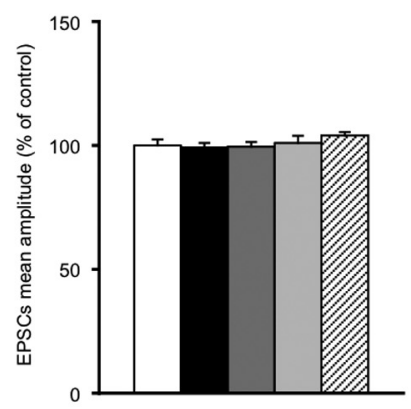

Figure 2. Pharmacological characterization of the presynaptic TRVP1 channel in the SON. $\boldsymbol{A}$, Representative example of the effect of capsaicin (10 $\mu \mathrm{M})$, a TRPV1 channel agonist, on mEPSCs. The holding potential was $-70 \mathrm{mV}$. Plots of frequency are single measurements, whereas plots of amplitude are mean \pm SEM over $30 \mathrm{~s}$. $\boldsymbol{B}$, Representative examples of the effect of selective TRPV1 channel blockers on mannitol-induced potentiation of mEPSCs. $\boldsymbol{a}$, Capsazepine (10 $\mu \mathrm{m})$; $\boldsymbol{b}$, SB366791 (100 $\mu \mathrm{m})$; and c, BCTC (10 $\mu \mathrm{M})$. C, Summary data for the effects of TRPV1 selective blockers. Frequency (left) and amplitude (right) of mEPSCs. No treatment $(n=7)$, mannitol $(n=7)$, mannitol plus capsazepine $(n=8)$, mannitol plus SB366791 $(n=7)$ and mannitol plus BCTC $(n=11)$. Data are mean \pm SEM. ${ }^{* *} p<0.01$, compared with no treatment.

SB366791 $(100 \mu \mathrm{M})$ or BCTC $(10 \mu \mathrm{M})$ without affecting the mean amplitude (SB366791: frequency $164 \pm 11 \%$ of control, $n$ $=7, p<0.01$, amplitude $101 \pm 2.9 \%$ of control, $n=7$; BCTC: frequency $163 \pm 8.4 \%$ of control, $n=11, p<0.01$, amplitude $104 \pm 1.4 \%$ of control, $n=11$ ) (Fig. $2 C$ ).

\section{Characterization of hyperosmotic stimulation- and} angiotensin II-induced potentiation of mEPSCs

Previous studies showed that endocannabinoids, whose receptors are localized on the presynaptic terminals, act as retrograde messengers and modulate synaptic activities in the SON (Hirasawa et al., 2004; Soya et al., 2005). To determine whether endocannabinoids, AVP, and OXT regulate synaptic transmission during hyperosmotic stimulation and angiotensin II application, we examined the effects of AM251 (CB1 receptor antagonist) (2 $\mu \mathrm{M}$ ), PMP-AVP (AVP V1a receptor antagonist) and atosiban (OXT receptor antagonist). Pre-exposure to AM251 significantly increased the frequency of mannitol- and angiotensin II-induced potentiation of mEPSCs (AM251 plus mannitol: frequency $237 \pm 14 \%$ of control, $n=8, p<0.05$ vs mannitol, amplitude $103 \pm 1.7 \%$ of control, $n=8$; AM251 plus angiotenisn II: frequency $209 \pm 21 \%$ of control, $n=8, p<0.05$ vs angiotensin II, amplitude $103 \pm 2.1 \%$ of control, $n=$ 8) (Fig. $3 A, B)$. Pre-exposure to PMP$\operatorname{AVP}(2 \mathrm{nM})$ and atosiban $(1 \mu \mathrm{M})$ did not affect mannitol- and angiotensin IIinduced potentiation of mEPSCs (PMPAVP plus mannitol: frequency $172 \pm 14 \%$ of control, amplitude $105 \pm 3.2 \%$ of control, $n=6$; PMP-AVP plus angiotensin II: frequency $155 \pm 15 \%$ of control, amplitude $99.1 \pm 2.0 \%$ of control, $n=4$; atosiban plus mannitol: frequency $179 \pm 12 \%$ of control, amplitude $104 \pm 2.1 \%$ of control, $n=6$; atosiban plus angiotensin II: frequency $142 \pm 5.3 \%$ of control, amplitude $104 \pm 1.6 \%$ of control, $n=6$ ) (Fig. 3B).

Since the AT1 receptor exists in glial cells, we also examined the effects of DL-fluorocitric acid (a glial metabolism inhibitor). Pretreatment with DL-fluorocitric acid for $150 \mathrm{~min}$ did not affect mannitol- and angiotensin II-induced potentiation of mEPSC (DL-fluorocitric plus mannitol: frequency $181 \pm 15 \%$ of control, amplitude $105 \pm 2.3 \%$ of control, $n=5$; DL-fluorocitric plus angiotensin II: frequency $143 \pm 11 \%$ of control, amplitude $97.4 \pm 1.8 \%$ of control, $n=4$ ) (Fig. $3 B$ ).

To examine whether the potentiation of mEPSC by mannitol is dependent on extracellular $\mathrm{Ca}^{2+}$, we used $\mathrm{Ca}^{2+}$-free solution. The frequency and amplitude of mEPSC in the $\mathrm{Ca}^{2+}$-free solution were significantly smaller than that in normal perfusion solution (normal vs $\mathrm{Ca}^{2+}$-free: frequency $0.79 \pm 0.1 \mathrm{~Hz}$ vs $0.69 \pm 0.1 \mathrm{~Hz}$, $n=8, p<0.05$, amplitude $27.7 \pm 0.7 \mathrm{pA}$ vs $23.5 \pm 0.2 \mathrm{pA}, n=8, p<0.01)$. Under this condition, mannitol did not increase the frequency and amplitude of mEPSC (frequency $106 \pm 6.3 \%$ of control, amplitude $101 \pm 1.3 \%$ of control, $n=8$ ) (Fig. 3C). Thus, the mannitol-induced potentiation of mEPSC was extracellular $\mathrm{Ca}^{2+}$-dependent.

Hyperosmotic stimulation and angiotensin II independently modulate mEPSCs through TRPV channels

We next examined whether angiotensin II and mannitol interact with each other, we used $10 \mu \mathrm{M}$ losartan, an AT1 receptor selective antagonist. Mannitol-induced potentiation of mEPSCs was not affected by pre-exposure to losartan (frequency $162 \pm$ $11 \%$ of control, $n=12, p<0.01$, amplitude $102 \pm 1.7 \%$ of control, $n=12$ ) (Fig. $4 A$ ). These results suggest that mannitol and angiotensin II independently modulate mEPSCs through 
TRPV channels and a lack of direct interaction between these two pathways.

Signal transduction by mannitol and angiotensin II in the presynaptic terminals of the SON

We examined the signal transduction activated by angiotensin II receptor in the presynaptic terminals of the SON by using a PKC inhibitor, bisindolylmaleimide I, and a calcium-ATPase inhibitor, thapsigargin. Bisindolylmaleimide I (10 nM) inhibited angiotensin II-induced potentiation of mEPSCs (frequency $105 \pm 4.0 \%$, amplitude $97.7 \pm 2.1 \%$ of control, $n=8$ ) (Fig. $4 B, C)$. On the other hand, thapsigargin $(10 \mathrm{nM})$ did not inhibit angiotensin II-induced potentiation of mEPSCs (frequency $187 \pm 19 \%$ of control, $n=5, p<$ 0.01 , amplitude $94.9 \pm 2.9 \%$ of control, $n$ $=5$ ) (Fig. 4C). These results suggest the involvement of PKC in signal transduction from the angiotensin II receptor to the TRPV channels. Interestingly, mannitol-induced potentiation of mEPSCs was not inhibited by bisindolylmaleimide I and thapsigargin (bisindolylmaleimide: frequency $195 \pm 12 \%$ of control, $n=4$, $p<0.01$, amplitude $102 \pm 2.1 \%$ of control, $n=4$; thapsigargin: frequency $191 \pm 14 \%$ of control, $n=4, p<0.01$, amplitude $104 \pm 1.8 \%$ of control, $n=4$ ) (Fig. 4C).

Effects of hyperosmotic stimulation and angiotensin II on mEPSCs in Trpv1 ${ }^{-/-}$mice

To determine whether the N-terminal variants of TRPV1 are required for the generation of the osmosensory response, we compared the effects of mannitol and angiotensin II on mEPSCs in the supraoptic MNCs of wild-type mice and $\operatorname{Trp} v 1^{-/-}$ mice (Fig. 5). The raw frequency and amplitude were not different between rats and mice. Mannitol- and angiotensin IIinduced increase in mEPSCs was significantly lower in $\operatorname{Tr} p v 1^{-1-}$ mice than in wild-type mice. However, the effects were not abolished completely even in $\operatorname{Trpv1} 1^{-/-}$ mice. The mean amplitude of mEPSCs was not affected in both wild-type and $\operatorname{Tr} p v 1^{-/-}$mice (wild-type vs $\operatorname{Tr} p v 1^{-/-}$; mannitol: frequency $191 \pm 16 \%(n=7)$ vs $119 \pm 4.0 \%(n=8)$ of control, $p<0.01$, amplitude $106 \pm 2.1 \%$ vs $101 \pm 2.1 \%$ of control; angiotensin II: $162 \pm 15 \%(n=6)$ vs $116 \pm 6.4 \%(n=7)$ of control, $p<0.01$, amplitude $101 \pm 2.6 \%$ vs $105 \pm 3.5 \%$ ).

\section{Discussion}

In the present study, mannitol-induced hyperosmotic stimulation and application of angiotensin II independently increased the frequency of mEPSCs without affecting their amplitude in supraoptic MNCs. Such potentiation of mEPSCs was enhanced
A

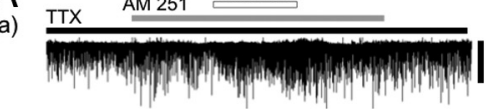

$40 \mathrm{pA}$
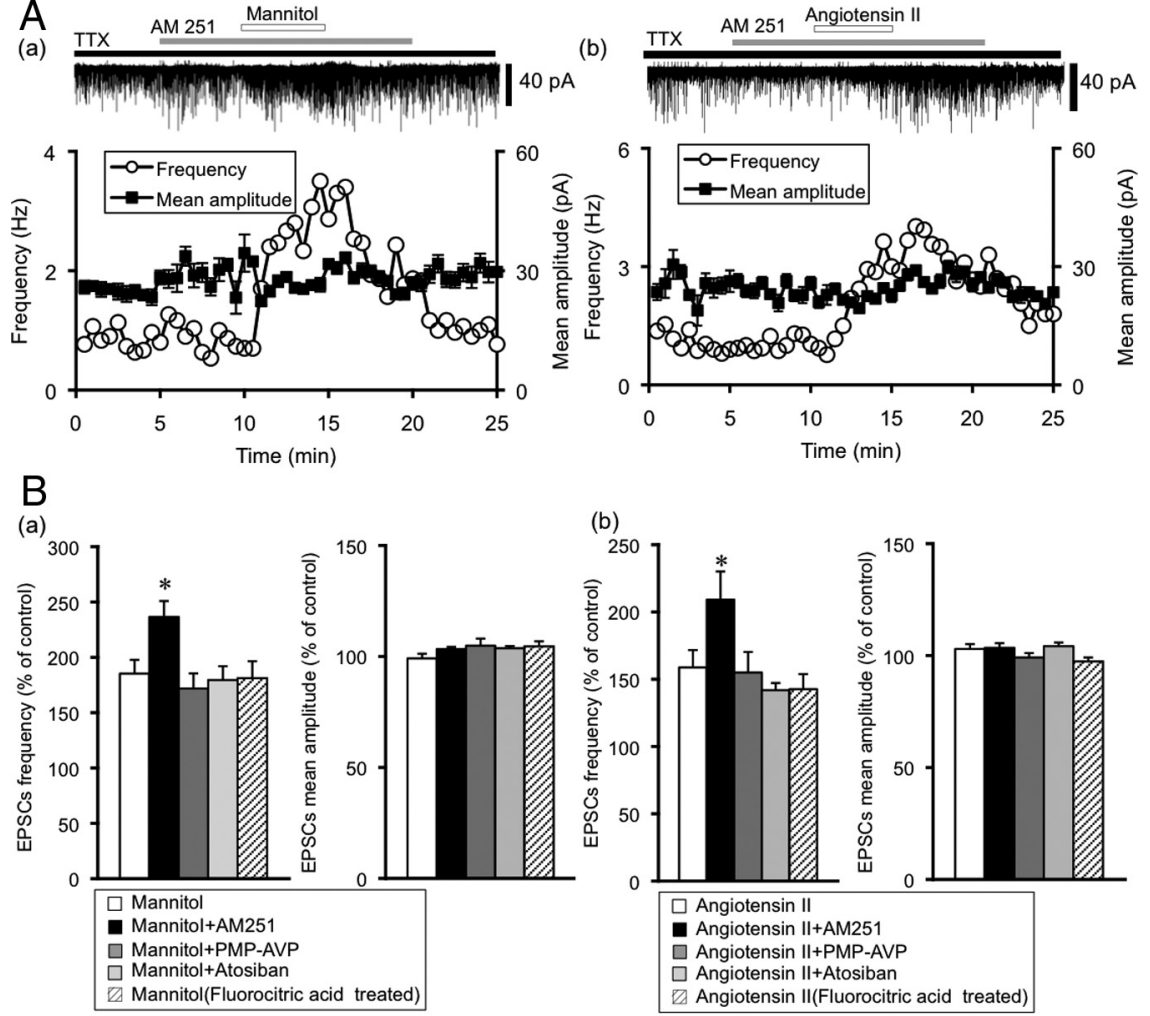

(a)

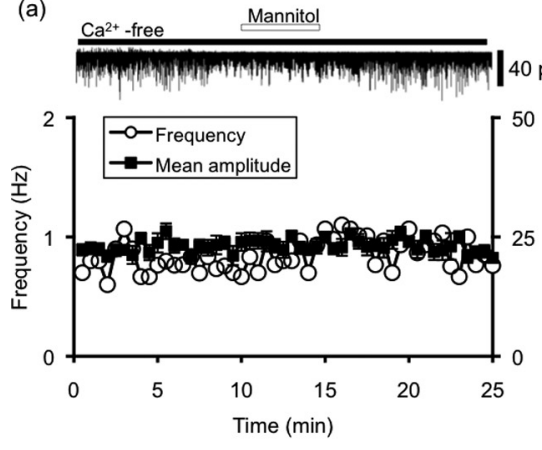

(b)

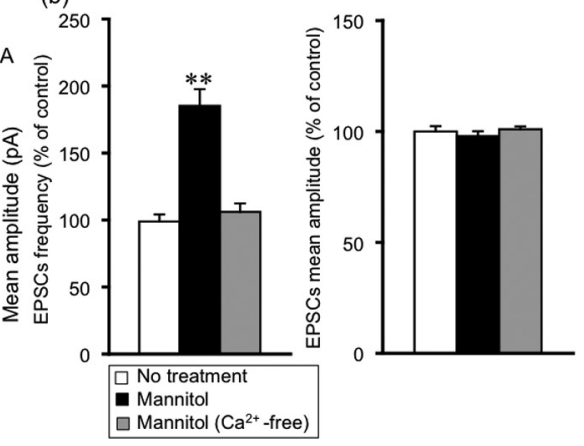

Figure 3. Characterization of the mannitol-and angiotensin Il-induced potentiation of mEPSCs. $A$, Representative examples of the effect of AM251, a cannabinoid type 1 receptor blocker, on the mannitol- and angiotensin II-induced potentiation of mEPSC. $\boldsymbol{a}$, Mannitol; $\boldsymbol{b}$, angiotensin II. The holding potential was $-70 \mathrm{mV}$. Plots of frequency are single measurements, whereas plots of amplitude are mean \pm SEM over 30 s. $\boldsymbol{B}$, Summary data for the characterization of mEPSCs under mannitol and angiotensin II application. $\boldsymbol{a}$, Frequency (left) and amplitude (right) of mEPSCs. mannitol $(n=8)$, mannitol plus AM251 $(n=8)$, mannitol plus $\operatorname{PMP}-\operatorname{AVP}(n=6)$, mannitol plus atosiban $(n=6)$ and mannitol (fluorocitric acid) $(n=5)$, respectively. $\boldsymbol{b}$, Angiotensin II $(n=8)$, angiotensin II plus AM251 $(n=6)$, angiotensin II plus PMP-AVP $(n=4)$, angiotensin II plus atosiban $(n=6)$ and angiotensin II (fluorocitric acid) $(n=4)$. Data are mean \pm SEM. ${ }^{*} p<0.05$, compared with mannitol or angiotensin II. C, Mannitol-induced potentiation of mEPSCs is extracellular $\mathrm{Ca}^{2+}$ dependent. $\boldsymbol{a}$, A representative example of the effect of mannitol $(60 \mathrm{~mm})$ on mannitol-induced potentiation of mEPSCs in the $\mathrm{Ca}^{2+}$-free perfusion medium. $\boldsymbol{b}$, Summary data for the effects of mannitol on frequency (left) and amplitude (right) of mEPSCs in normal solution $(n=8)$ and in $\mathrm{Ca}^{2+}$-free solution $(n=8)$. Data are mean \pm SEM. ${ }^{* *} p<0.01$, compared with no treatment.

by pre-exposure to AM251, a cannabinoid type1 receptor blocker, and attenuated by pre-exposure to ruthenium red, a nonspecific TRPV channel blocker. In the $\operatorname{Tr} p v 1^{-1-}$ mice, the effects of mannitol and angiotensin II were markedly attenuated compared with those in wild-type mice.

We have reported that application of mannitol or angiotensin II modulated mEPSCs by acting on the presynaptic terminals in the SON (Inenaga et al., 1997; Ozaki et al., 2004). The present results also support these conclusions because (1) recordings were conducted from neurons within thin slices containing only 
A

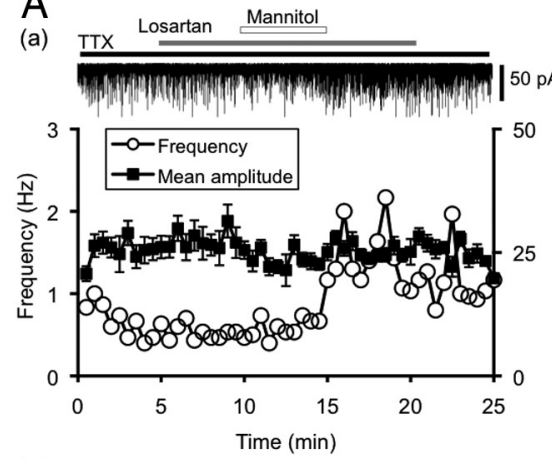

B

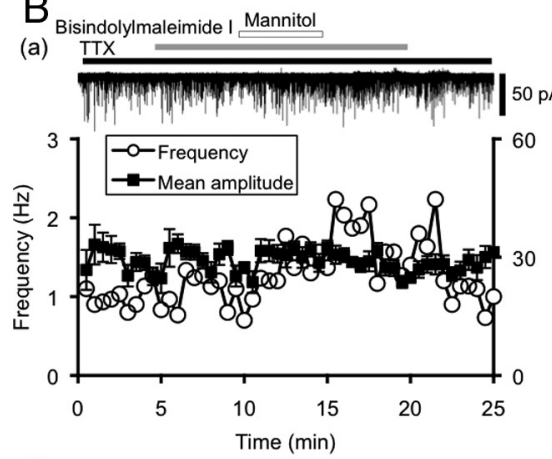

C

$$
\text { (a) }
$$
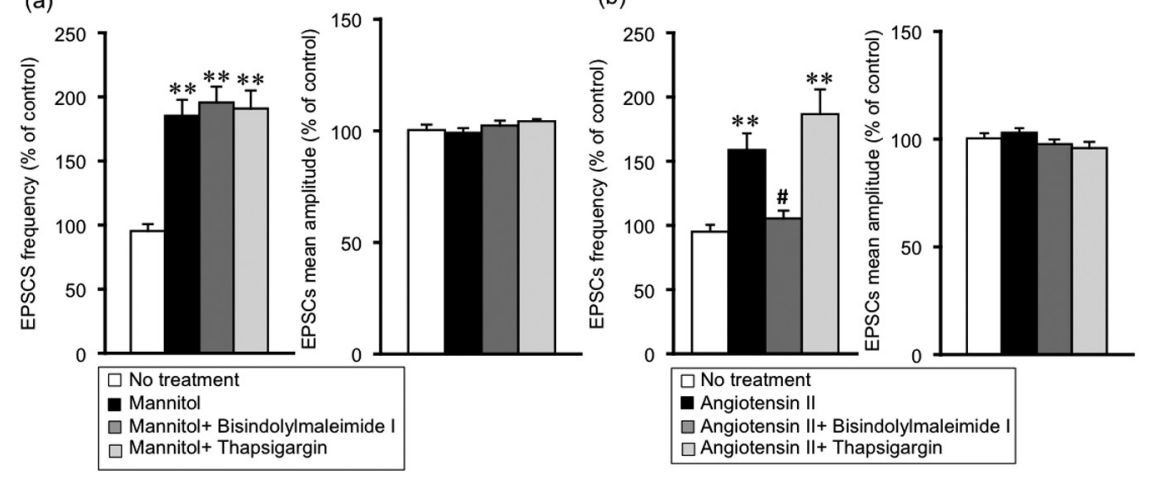

Figure 4. Mannitol and angiotensin Il independently modulate mEPSCs through PKC pathway and TRPV channels. $A$, Mannitolinduced potentiation of mEPSCs is not affected by pre-exposure to losartan, angiotensin II type 1 receptor antagonist. $\boldsymbol{a}$, Representative example of the effect of losartan $(10 \mu \mathrm{m})$ on mannitol-induced potentiation of mEPSCS. The holding potential was -70 $\mathrm{mV}$. Plots of frequency are single measurements, whereas plots of amplitude are mean \pm SEM over $30 \mathrm{~s}$. $\boldsymbol{b}$, Summary data for the effects of mannitol on the frequency and amplitude of $\mathrm{mEPSC}$ in the presence of losartan $(n=12)$. Data are mean \pm SEM. ${ }^{* *} p<$ 0.01, compared with no treatment. $\boldsymbol{B}$, Representative examples of the effect of bisindolylmaleimide I (PKC inhibitor) on mannitol (a) - and angiotensin II (b)-induced potentiation of mEPSCS. C, Summary data for the effects of bisindolylmaleimide I and thapsigargin (calcium-ATPase inhibitor) on mannitol- and angiotensin II-induced potentiation of mEPSCs. $\boldsymbol{a}$, Frequency (left) and amplitude (right) of mEPSCs. No treatment $(n=7)$, mannitol $(n=8)$, bisindolylmaleimide I (10 nM) $(n=4)$, thapsigargin (10 nм) $(n=4)$. $\boldsymbol{b}$, Frequency (left) and amplitude (right) of mEPSCs. No treatment $(n=7)$, angiotensin II $(n=8)$, bisindolylmaleimide I $(n=7)$, thapsigargin $(n=5)$. Data are mean \pm SEM. ${ }^{* *} p<0.01$, compared with no treatment. ${ }^{*} p<0.01$, compared with mannitol or angiotensin II.

SON and the perinuclear zone, (2) the mannitol- and angiotensin II-induced increase in frequency of mEPSCs was observed even in the presence of TTX without affecting the amplitude of the mEPSCs, and (3) the presence of BAPTA in the pipette solution did not affect the potentiation of mEPSCs induced by mannitol and angiotensin II. The mEPSCs in the present study thus reflect spontaneous transmitter release from the terminals of cut axons, disconnected from their cell bodies, in response to application of mannitol and angiotensin II.

Ruthenium red attenuated the mannitol- and angiotensin IIinduced increase of mEPSC frequency without affecting mEPSC amplitude. Since ruthenium red is a nonspecific TRPV channels blocker, these results suggest that TRPV channels mediate the potentiation of mEPSCs induced by mannitol and angiotensin II. The TRPV channels have been shown to play a role in mechanosensation and osmosensation. The osmosensitivity in the SON is considerably mediated by the osmosensory transduction channel, which is activated by hyperosmotic stimulation and inhibited by hypo-osmotic stimulation (Oliet and Bourque, 1993a,b, 1996). A recent study has demonstrated that an N-terminal splice variant of TRPV1 (N-truncated form of the TRPV1) is expressed on the supraoptic MNCs and is essential for osmosensory transduction in the SON (Sharif Naeini et al., 2006). The N-terminal end of TRPV1 is known to be a capsaicin receptor (Caterina et al., 1997), and parts of the $\mathrm{N}$ terminus of the protein are required to mediate the effects of capsaicin (Schumacher et al., 2000; Jung et al., 2002; Wang et al., 2004; Hellwig et al., 2005; Lu et al., 2005; Vos et al., 2006). Thus, the $\mathrm{N}$-truncated form of the TRPV1 is not sensitive to capsaicin in the supraoptic MNCs, and $\operatorname{trp} v 1^{-/-}$mice had impaired AVP secretion in response to synaptic hyperosmolality and infusion of angiotensin II (Sharif Naeini et al., 2006). The present study is in good agreement with these results except that the site of action was presynaptic terminals, because capsaicin did not have a significant effect on mEPSCs, and mannitol-induced potentiation of mEPSCs could not be attenuated by the TRPV1 selective blocker capsazepine. Although we did not identify the N-terminal splice variant of TRPV1 by a specific antibody, the results using $\operatorname{Tr} p v 1^{-/-}$mice strongly support the presence of the $\mathrm{N}$-truncated form of TRPV1 in the presynaptic terminals. However, TRPV1 is required for intrinsic osmoreception in the OVLT that projects its axon into the SON and is very sensitive to systemic osmotic changes (Ciura and Bourque, 2006). Moreover, in the paraventricular nucleus (PVN), activation of presynaptic TRPV1 by capsaicin excites spinally projecting PVN neurons through increase of mEPSC frequency without affecting the amplitude, a mechanism very similar to that of the present results ( $\mathrm{Li}$ et al., 2004). At present, the reason for the discrepancy, that is the regulatory role of $\mathrm{N}$-truncated form of the TRPV1 versus TRPV1, is not clear. Since the TRPV1 is widely present in the brain and has many splice variants, the mechanism of regulation may vary depending on the expression of the variant form.

In our experiments using $\operatorname{Trp} v 1^{-1-}$ mice, potentiation of mEPSCs induced by mannitol and angiotensin II was markedly reduced compared with the wild-type. However, it was not com- 
plete and the possible involvement of other TRP channels or mechanisms could not be excluded. In fact, the TRPV4 channel is reported to function as an osmosensor in the SON (Liedtke et al., 2000; Strotmann et al., 2000, Liedtke and Friedman, 2003; Mizuno et al., 2003), although this channel is reported to be activated by cell swelling and is insensitive to hyperosmotic stimulation (Liedtke et al., 2000; Strotmann et al., 2000; Alessandri-Haber et al., 2003). Extracellular matrices around the MNCs, such as protein, other mechanochannels and other TRP channels, such as TRPC, may participate in mechanical regulation and glutamatergic release.

Interestingly, pre-exposure to AM251 potentiated mEPSCs induced by mannitol and angiotensin II. These results suggest that endocannabinoids modulate the excitatory synaptic inputs. Various endogenous factors regulate the release of transmitters in the SON. We have demonstrated previously that cannabinoids modulate synaptic activity in the rat SON (Soya et al., 2005). Recent studies have demonstrated also that synaptic transmission in the SON is regulated by endocannabinoids (Hirasawa et al., 2004; McDonald et al., 2008). In high-salt diet rats, endocannabinoids regulate blood pressure through activation of the TRPV1 channel (Wang et al., 2007). Considered collectively, these results suggest that endocannabinoids participate in osmoregulation and interact with the TRP channels.

Since the potentiation of mEPSCs was induced by both mannitol and angiotensin II and suppressed by pre-exposure to ruthenium red, we examined whether there is an interaction between these two pathways. Pre-exposure to losartan, an AT1 receptor antagonist, had no effect on mannitol-induced potentiation of mEPSCs. Mannitol-induced potentiation of mEPSCs was not suppressed by bisindolylmaleimide I or thapsigargin. Moreover, ruthenium red does not easily cross the cell membrane and commonly has to be dialyzed into cells to modulate mitochondrial calcium transport (Tang and Zucker, 1997). Thus, the effects of ruthenium red on mannitol-induced currents are more likely to be due to channel blockade rather than via intracellular signal transduction.

To date, four distinct AT receptors have been identified (AT1, AT2, AT3 and AT4) (Unger et al., 1996). The AT1 receptor is predominant in the SON (Culman et al., 1995). However, the mechanism through which AT1 receptor activation leads to potentiation of mEPSCs presynaptically remains elusive. The AT1 receptor is a G-protein coupled receptor, which is responsible for most of the physiological actions of angiotensin II (Conchon et al., 1998). Signal transduction via AT1 receptor follows the Gq protein pathway. Activated AT1 receptors are coupled to the Gq protein and they increase phospholipase C (PLC) activity, resulting in the cleavage of phosphatidylinositol-4,5-bisphosphate $\left(\mathrm{PIP}_{2}\right)$ by PLC, yielding two second messengers: inositol-1,4,5trisphosphate $\left(\mathrm{IP}_{3}\right)$ and diacylglycerol (DAG). DAG with calcium activates $\mathrm{PKC}$, and $\mathrm{IP}_{3}$ activates calcium release channels in en-
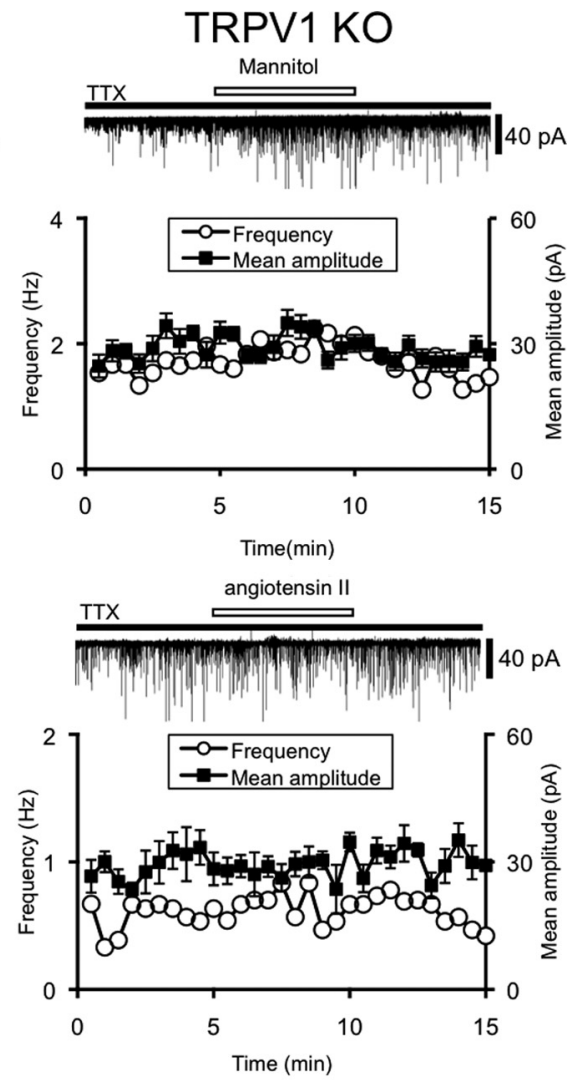

Figure 5. Effects of mannitol and angiotensin II on mEPSCs in the SON of wild-type and Trpv1 ${ }^{-1-}$ mice. Representative Trpv $1^{-1-}$ mice (TRPV1KO). All experiments were recorded in the presence of TTX (1 $\left.\mu \mathrm{M}\right)$. The holding potential was $-70 \mathrm{mV}$ Plots of frequency are single measurements, whereas plots of amplitude are mean \pm SEM over $30 \mathrm{~s}$.

doplasmic reticulum membranes. We examined the signal transduction activated by angiotensin II. Angiotensin II-induced increase of mEPSC frequency was suppressed by pre-exposure to the PKC inhibitor (bisindolylmaleimide I) but not by the intracellular calcium-ATPase inhibitor (thapsigargin). These results suggest that angiotensin II-induced potentiation of mEPSCs is mediated through the TRPV channels by activating the AT1 receptor, Gq-PLC-DAG-PKC pathway. Interestingly, activation of PKC is also involved in the TRPV1-mediated potentiation of mEPSCs at the presynaptic terminals of sensory neurons, which contributes to the pathophysiology of chronic pain conditions (Sikand and Premkumar, 2007).

\section{Limitation of the study}

In the present study, $\sim 80 \%$ of the tested supraoptic MNCs were sensitive to mannitol and angiotensin II. Supraoptic MNCs (AVP neurons or OXT neurons) are divided into two groups based on their electrophysiological characteristics. By combining immunohistochemical and electrophysiological techniques, most of the phasically firing neurons contain AVP (phasic neurons) whereas the other neurons that do not fire phasically (nonphasic neurons) contain OXT (Yamashita et al., 1983; Cobbett et al., 1986). Subsequent studies revealed that the majority of OXT neurons displayed a sustained outward rectification at depolarized potentials and hyperpolarization-induced inward rectification; those were largely absent in AVP neurons (Armstrong et al., 1994; Stern and Armstrong, 1995; Hirasawa et al., 2003). Although we did not identify the cell types electrophysiologically in the present study, the possibility that the actions of mannitol and angiotensin 
II are limited to a single cell type (AVP neurons or OXT neurons) is unlikely. A previous immunohistochemical study demonstrated that AVP neurons are more common in the caudal and ventral parts of the SON, while OXT neurons tend to be found rostrally and dorsally (Rhodes et al., 1981). Subsequent topographic analysis revealed the majority of Fos-expressing AVP neurons occupy the ventral part of the SON, while Fos-OXT neurons are located mainly in the dorsal part on hyperosmotic stimulation (Pirnik et al., 2004). Since we recorded mEPSCs in the ventral part of the SON, this methodological approach might affect the proportion of angiotensin II-sensitive MNCs.

In conclusion, mannitol-induced hyperosmotic stimulation and application of angiotensin II independently increased the frequency of mEPSCs through capsaicin-insensitive TRPV1 channels in the presynaptic terminals of the SON. Mannitol might act on the TRPV1 channels directly, while angiotensin II acts via AT1 receptor and signal transduction of the Gq-PLCDAG-PKC pathway.

\section{References}

Alessandri-Haber N, Yeh JJ, Boyd AE, Parada CA, Chen X, Reichling DB, Levine JD (2003) Hypotonicity induces TRPV4-mediated nociception in rat. Neuron 39:497-511.

Armstrong WE, Smith BN, Tian M (1994) Electrophysiological characteristics of immunochemically identified rat oxytocin and vasopressin neurones in vitro. J Physiol 475:115-128.

Bourque CW (1989) Ionic basis for the intrinsic activation of rat supraoptic neurones by hyperosmotic stimuli. J Physiol 417:263-277.

Bourque CW, Oliet SH, Richard D (1994) Osmoreceptors, osmoreception, and osmoregulation. Front Neuroendocrinol 15:231-274.

Caterina MJ, Schumacher MA, Tominaga M, Rosen TA, Levine JD, Julius D (1997) The capsaicin receptor: a heat-activated ion channel in the pain pathway. Nature 389:816-824.

Caterina MJ, Leffler A, Malmberg AB, Martin WJ, Trafton J, Petersen-Zeitz KR, Koltzenburg M, Basbaum AI, Julius D (2000) Impaired nociception and pain sensation in mice lacking the capsaicin receptor. Science 288:306-313.

CiuraS, Bourque CW (2006) Transient receptor potential vanilloid 1 is required for intrinsic osmoreception in organum vasculosum lamina terminalis neurons and for normal thirst responses to systemic hyperosmolality. J Neurosci 26: 9069-9075.

Cobbett P, Smithson KG, Hatton GI (1986) Immunoreactivity to vasopressin- but not oxytocin-associated neurophysin antiserum in phasic neurons of rat hypothalamic paraventricular nucleus. Brain Res 362:7-16.

Conchon S, Peltier N, Corvol P, Clauser E (1998) A noninternalized nondesensitized truncated AT1A receptor transduces an amplified ANG II signal. Am J Physiol 274:E336-E345.

Culman J, Höhle S, Qadri F, Edling O, Blume A, Lebrun C, Unger T (1995) Angiotensin as neuromodulator/neurotransmitter in central control of body fluid and electrolyte homeostasis. Clin Exp Hypertens 17:281-293.

Hellwig N, Albrecht N, Harteneck C, Schultz G, Schaefer M (2005) Homoand heteromeric assembly of TRPV channel subunits. J Cell Sci 118:917-928.

Hirasawa M, Mouginot D, Kozoriz MG, Kombian SB, Pittman QJ (2003) Vasopressin differentially modulates non-NMDA receptors in vasopressin and oxytocin neurons in the supraoptic nucleus. J Neurosci 23:4270-4277.

Hirasawa M, Schwab Y, Natah S, Hillard CJ, Mackie K, Sharkey KA, Pittman QJ (2004) Dendritically released transmitters cooperate via autocrine and retrograde actions to inhibit afferent excitation in rat brain. J Physiol 559:611-624.

Inenaga K, Cui LN, Nagatomo T, Honda E, Ueta Y, Yamashita H (1997) Osmotic modulation in glutamatergic excitatory synaptic inputs to neurons in the supraoptic nucleus of rat hypothalamus in vitro. J Neuroendocrinol 9:63-68.

Jung J, Lee SY, Hwang SW, Cho H, Shin J, Kang YS, Kim S, Oh U (2002) Agonist recognition sites in the cytosolic tails of vanilloid receptor 1.J Biol Chem 277:44448-44454.

Kabashima N, Shibuya I, Ibrahim N, Ueta Y, Yamashita H (1997) Inhibition of spontaneous EPSCs and IPSCs by presynaptic GABAB receptors on rat supraoptic magnocellular neurons. J Physiol 504:113-126.

Leng G, Mason WT, Dyer RG (1982) The supraoptic nucleus as an osmoreceptor. Neuroendocrinology 34:75-82.

Li DP, Chen SR, Pan HL (2004) VR1 receptor activation induces glutamate release and postsynaptic firing in the paraventricular nucleus. J Neurophysiol 92:1807-1816.

Liedtke W, Friedman JM (2003) Abnormal osmotic regulation in trpv4-/mice. Proc Natl Acad Sci U S A 100:13698-13703.

Liedtke W, Choe Y, Martí-Renom MA, Bell AM, Denis CS, Sali A, Hudspeth AJ, Friedman JM, Heller S (2000) Vanilloid receptor-related osmotically activated channel (VR-OAC), a candidate vertebrate osmoreceptor. Cell 103:525-535.

Lu G, Henderson D, Liu L, Reinhart PH, Simon SA (2005) TRPV1b, a functional human vanilloid receptor splice variant. Mol Pharmacol 67:11191127.

Mason WT (1980) Supraoptic neurones of rat hypothalamus are osmosensitive. Nature 287:154-157.

McDonald NA, Kuzmiski JB, Naderi N, Schwab Y, Pittman QJ (2008) Endogenous modulators of synaptic transmission: cannabinoid regulation in the supraoptic nucleus. Prog Brain Res 170:129-136.

Mizuno A, Matsumoto N, Imai M, Suzuki M (2003) Impaired osmotic sensation in mice lacking TRPV4. Am J Physiol Cell Physiol 285:C96-C101.

Nagatomo T, Inenaga K, Yamashita H (1995) Transient outward current in adult rat supraoptic neurones with slice patch-clamp technique: inhibition by angiotensin II. J Physiol 485:87-96.

Okuya S, Inenaga K, Kaneko T, Yamashita H (1987) Angiotensin II sensitive neurons in the supraoptic nucleus, subfornical organ and anteroventral third ventricle of rats in vitro. Brain Res 402:58-67.

Oliet SH, Bourque CW (1992) Properties of supraoptic magnocellular neurones isolated from the adult rat. J Physiol 455:291-306.

Oliet SH, Bourque CW (1993a) Mechanosensitive channels transduce osmosensitivity in supraoptic neurons. Nature 364:341-343.

Oliet SH, Bourque CW (1993b) Steady-state osmotic modulation of cationic conductance in neurons of rat supraoptic nucleus. Am J Physiol 265:R1475-R1479.

Oliet SH, Bourque CW (1996) Gadolinium uncouples mechanical detection and osmoreceptor potential in supraoptic neurons. Neuron 16:175-181.

Ozaki Y, Soya A, Nakamura J, Matsumoto T, Ueta Y (2004) Potentiation by angiotensin II of spontaneous excitatory postsynaptic currents in rat supraoptic magnocellular neurones. J Neuroendocrinol 16:871-879.

Pirnik Z, Mravec B, Kiss A (2004) Fos protein expression in mouse hypothalamic paraventricular (PVN) and supraoptic (SON) nuclei upon osmotic stimulus: colocalization with vasopressin, oxytocin, and tyrosine hydroxylase. Neurochem Int 45:597-607.

Rhodes CH, Morrell JI, Pfaff DW (1981) Immunohistochemical analysis of magnocellular elements in rat hypothalamus: distribution and numbers of cells containing neurophysin, oxytocin, and vasopressin. J Comp Neurol 198:45-64.

Richard D, Bourque CW (1995) Synaptic control of rat supraoptic neurones during osmotic stimulation of the organum vasculosum lamina terminalis in vitro. J Physiol 489:567-577.

Schumacher MA, Moff I, Sudanagunta SP, Levine JD (2000) Molecular cloning of an $\mathrm{N}$-terminal splice variant of the capsaicin receptor. Loss of $\mathrm{N}$-terminal domain suggests functional divergence among capsaicin receptor subtypes. J Biol Chem 275:2756-2762.

Sharif Naeini R, Witty MF, Séguéla P, Bourque CW (2006) An N-terminal variant of Trpvl channel is required for osmosensory transduction. Nat Neurosci 9:93-98.

Sikand P, Premkumar LS (2007) Potentiation of glutamatergic synaptic transmission by protein kinase C-mediated sensitization of TRPV1 at the first sensory synapse. J Physiol 581:631-647.

Soya A, Serino R, Fujihara H, Onaka T, Ozaki Y, Saito T, Nakamura J, Ueta Y (2005) Cannabinoids modulate synaptic activity in the rat supraoptic nucleus. J Neuroendocrinol 17:609-615.

Stachniak TJ, Bourque CW (2006) Visually guided whole cell patch clamp of mouse supraoptic nucleus neurons in cultured and acute conditions. Am J Physiol Regul Integr Comp Physiol 291:R68-R76.

Stern JE, Armstrong WE (1995) Electrophysiological differences between oxytocin and vasopressin neurones recorded from female rats in vitro. J Physiol 488:701-708. 
Strotmann R, Harteneck C, Nunnenmacher K, Schultz G, Plant TD (2000) OTRPC4, a nonselective cation channel that confers sensitivity to extracellular osmolarity. Nat Cell Biol 2:695-702.

Tang Y, Zucker RS (1997) Mitochondrial involvement in post-tetanic potentiation of synaptic transmission. Neuron 18:483-491.

Unger T, Chung O, Csikos T, Culman J, Gallinat S, Gohlke P, Hohle S, Meffert S, Stoll M, Stroth U, Zhu YZ (1996) Angiotensin receptors. J Hypertens (Suppl 14):S95-S103.

Vos MH, Neelands TR, McDonald HA, Choi W, Kroeger PE, Puttfarcken PS, Faltynek CR, Moreland RB, Han P (2006) TRPV1b overexpression negatively regulates TRPV1 responsiveness to capsaicin, heat and low $\mathrm{pH}$ in HEK293 cells. J Neurochem 99:1088-1102.
Wang C, Hu HZ, Colton CK, Wood JD, Zhu MX (2004) An alternative splicing product of the murine trpvl gene dominant negatively modulates the activity of TRPV1 channels. J Biol Chem 279:37423-37430.

Wang Y, Kaminski NE, Wang DH (2007) Endocannabinoid regulates blood pressure via activation of the transient receptor potential vanilloid type 1 in Wistar rats fed a high-salt diet. J Pharmacol Exp Ther 321:763-769.

Yamashita H, Inenaga K, Kawata M, Sano Y (1983) Phasically firing neurons in the supraoptic nucleus of the rat hypothalamus: immunocytochemical and electrophysiological studies. Neurosci Lett 37:87-92.

Yang CR, Phillips MI, Renaud LP (1992) Angiotensin II receptor activation depolarizes rat supraoptic neurons in vitro. Am J Physiol 263:R1333R1338. 